\title{
The Next Step
}

Dear Reader,

Engine developments based on a holistic approach create considerable added value. First, it is easier to leverage the potential of the individual components in an integrated system - the result is more than just the sum of all the parts of the engine. Second, the OEM benefits from a coordinated, internally optimized and verified overall solution that it gets, so to speak, as a turnkey system, thus saving considerable effort in developing and integrating the powertrain into its application - including the ever more complex exhaust system. While the effort is now an issue for the engine manufacturer, it does have the opportunity to exploit development synergies, resulting in cost savings; the OEMs and their customers the operators - ultimately benefit from this. The latter can also outsource the task of maintenance to the engine manufacturer, resulting in a classic win-win situation: one side can expand its business while the other side saves time, effort and thus costs. We can assume that systems competence will in future become a greater core success factor for engine manufacturers than it is today.

However, clear overall system expertise is the basic prerequisite and thus the enabler of greater electrification of the powertrain through hybridization. In light of the current environmental political debate around the world, a number of concepts have already been presented in which applications have been electrified and hybridized. In the coming weeks and months, we at MTZindustrial will also be engaging more closely with these developments and reporting on the latest research and development findings. In the latest issue, however, our cover story first looks into systems engineering of the combustion engine. I hope you enjoy reading about it.
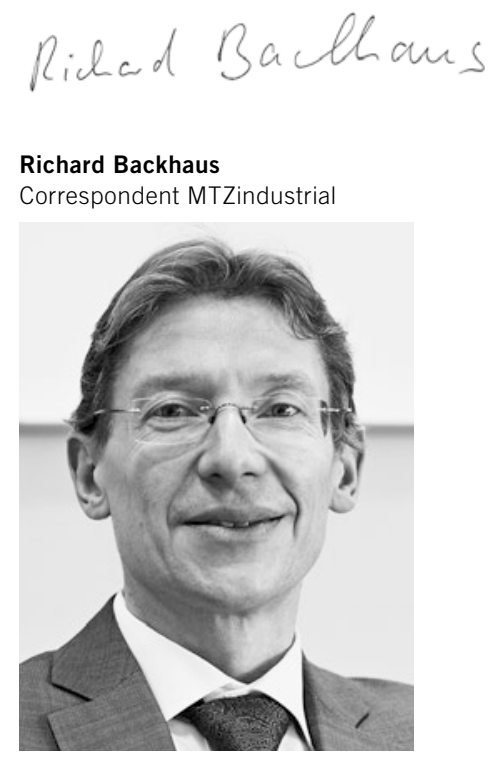